\title{
Effects of Collagen Nanofibrils on Turbid Water
}

\author{
Nicole Aylmer \\ Chemical Engineering, \\ Manhattan College \\ New York City, USA
}

\author{
Amanda Belluccio \\ Chemical Engineering, \\ Manhattan College \\ New York City, USA
}

\author{
Gennaro Maffia \\ Chemical Engineering, \\ Manhattan College \\ New York City, USA
}

\begin{abstract}
Having clean water is a problem throughout the whole world. The purpose of this paper is to test different amounts and concentrations of collagen dispersions in turbid water and use different methods to see which is more efficient in cleaning the water. We studied two different experiments, one using a shake and settle method and one using a refrigerated centrifuge. We found that the refrigerated centrifuge produced lower NTU values than the shake and settle method. This conclusion means that the refrigerated centrifuge cleaned the turbid water more effectively than shaking a sample cell and letting it settle on its own.
\end{abstract}

\section{INTRODUCTION}

According to Maffia's research (Maffia), collagen is made up of nanofibrils that can hold almost 500 times its weight in water. These fibers promote the agglomeration of suspended particles in water. As the particles agglomerate, flocs are formed. When compared to smaller particles, these flocs have a faster settling time, thus making it easier to filter out of water. The experiment was conducted using previously prepared collagen dispersions following Maffia's patent.

Before performing this experiment we realized that obtaining clean water is an ongoing issue throughout the world. Additionally, past methods of cleaning turbid water prove to be detrimental to humans and the environment. According to Maffia's patent, it was discovered that, "settling rates are more than ten times faster with collagen than with conventional chemical treatment (Maffia).." Not only does collagen prove to be more effective in settling particulates, it is also a safer alternative than chemical treatment as it is a biological material found in the human body in connective tissue and not harmful to humans or the environment.

\section{A. Collagen Research Group}

The collagen research began in the early 1980s at Dartmouth College (ref). It began as a response to the need within the cell culture scientific community for platforms, which were being utilized for immobilizing Chinese Hamster Ovary cells (CHO) among others. A group of interested researcher organized to share collagen nanofibrils and develop new applications.

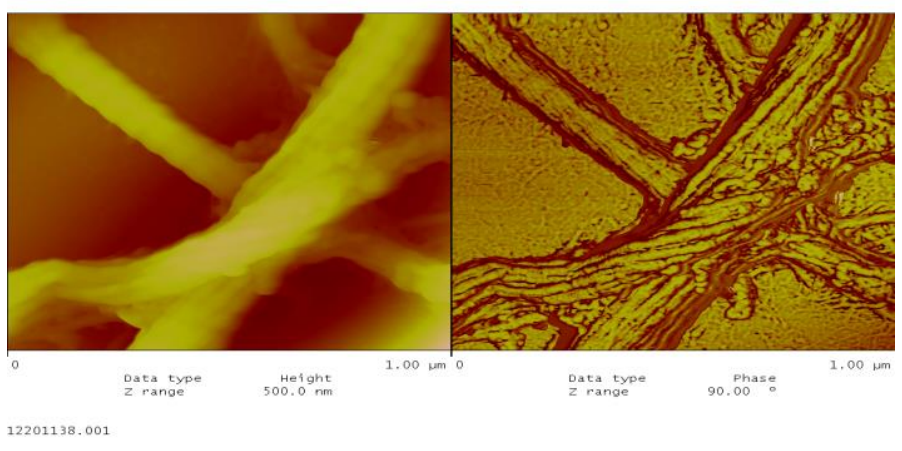

Collagen nanofibrils are about $50 \mathrm{~nm}$ in diameter and several hundred nm long. An atomic force micrograph is shown in Fig. 1 above.

Thus the Collagen Research Group, CRG, was formed. It continued in industry at Verax, Inc. a spin-off of the original Dartmouth work., until about 1995. Concurrently, the CRG

The focus of this current work is to use collagen nanofibrils to disrupt the colloidal nature of contaminated water, causing flocculation and settling of contaminants.

\section{MATERIALS AND METHODS}

In moving forward in this experiment we wanted to find the most efficient way of decreasing the turbidity in contaminated water.

Two different methods, shake and settle and centrifuging were devised for the purposes of this experiment. For the shake and settle method, we tested four different percentages of collagen dispersions $(0 \%, 0.3 \%$, $0.6 \%, 0.9 \%$ ) with different dosing amounts in the turbid water.

The formula for the collagen dispersions is generically stated as:

Collagen nanofibrils

Weak Organic Acid Water (deionized)
$\mathrm{X} \%$

$5 \%$ in the case of acetic acid

$100 \%-5 \%-X$ 
Blending of these materials requires about 10 minutes in a high shear blender. The resulting material exhibits non-Newtonian behavior as well as time dependency. The rheology of this material is the subject of a recent report by Sandy Shivakumar.

\section{A. Current Experiment}

A stock solution of "mock" contaminated water was prepared using kaolin. Every ten minutes, we recorded a reading from the turbidimeter of each sample cell. This was repeated for an hour only shaking the sample cell initially and letting it settle on its own. For the centrifuge, we tested the same four percentages of collagen at different amounts of drops in the turbid water just like the shake and settle method. We used a refrigerated centrifuge at 5 degrees Celsius and a speed of $3000 \mathrm{rpm}$ to force the collagen and kaolin to interact and eventually settle out. We recorded a reading off the turbidimeter of each sample cell again, but for five different amounts of time instead of every ten minutes for an hour.

This experiment was performed to test which dispersions of collagen would cause better settling and in the end a lower turbidity. The two methods previously explained were chosen to test the most efficient and cost effective way to distribute the collagen. If collagen dispersions are added to the water then the kaolin will conglomerate and settle out, causing the turbidity to decrease. Additionally adding more drops of collagen with a higher concentration will yield a lower turbidity. Centrifuging will cause too much disruption in the water, stopping the collagen fibers from interacting well with the kaolin particles. However, centrifuging for longer periods of time will increase the contact time between the kaolin particles and collagen fibers, yielding a lower turbidity reading.

\section{MATERIALS}

- Collagen nanofibrils.

- Pipettes.

- Hach 2100 Q IS Portable Turbidity Meter.

- Hach Sample Cell for 2100 Portable Turbidity Meter.

- Beckman Coulter Allegra 6R Refrigerated Centrifuge.

- Fisherbrand Easy Reader Polypropylene Centrifuge Tubes.

- Collagen dispersions at $0.3 \%, 0.6 \%$, and $0.9 \%$.

- Kaolin powder.

- Plastic spoon to measure kaolin.

- Intell-Lab Intelligent Precision Toploading.

- Denver Instrument Company TR Series Toploading Balance

- Deionized water.

\section{METHODS}

To set up this experiment, gather premade collagen dispersions formulated based on Dr. Maffia's patent. Then, use the Hach 2100 Q IS Portable Turbidity Meter and Denver Instrument Company TR Series Toploading Balance to find out how much kaolin needs to be added to $15 \mathrm{~mL}$ of deionized water to produce a target of around 1000 NTU.

\section{A. Shake and Settle:}

Initially, one needs to find a reading for the kaolin/water solution without adding any collagen. To do this, add $15 \mathrm{~mL}$ of deionized water and the sample weight of kaolin to a sample cell and, using the shake and settle method, shake ten times before checking the reading off of the turbidity meter. Then, leave the sample cell on the table and check the reading every ten minutes for an hour to see how much the kaolin settles. Record the turbidity value at ten-minute increments.

Now, one must test different amounts of different percentages of collagen mixed with the kaolin/water solution using the shake and settle method. First, start with the $0.6 \%$ collagen and test $1,2,3,4$, and 5 drops for an hour. Measure the weight of each drop and find the ppm. To find the ppm, multiply the weight of the drop by the percentage of collagen one is using, then divide by 10 and multiply by $10^{\wedge} 6$. Let the solution in the sample cell sit on the table and check the reading every ten minutes for an hour using the turbidity meter.

Next, use the $0.9 \%$ collagen and test $1,2,3,4$, and 5 drops for an hour. Measure the weight of each drop and find the ppm using the method mentioned before. Let the solution in the sample cell sit on the table and check the reading every ten minutes for an hour using the turbidity meter.

Then, use the $0.3 \%$ collagen and test $1,2,3,4$, and 5 drops for an hour. Measure the weight of each drop and find the ppm using the method mentioned before. Let the solution in the sample cell sit on the table and check the reading every ten minutes for an hour using the turbidity meter.

\section{B. Refrigerated Centrifuge:}

As in the shake and settle method, initially, one needs to find a reading for the kaolin/water solution without adding any collagen. To do this, add $15 \mathrm{~mL}$ of deionized water and the sample weight of kaolin to a sample cell and, using the refrigerated centrifuge method, test at $0,3,5,10$, and 15 minutes before checking the reading off of the turbidity meter.

Now, one must test different amounts of different percentages of collagen mixed with the appropriate weight of kaolin and use a refrigerated centrifuge at 5 degrees Celsius to mix the kaolin/collagen solution at 3000 rpm. First, start with $0.3 \%$ collagen and test $1,2,3,4$, and 5 drops at $0,3,5,10$, and 15 minutes in the refrigerated centrifuge. Measure the weight of each drop and find the ppm. To find the ppm, multiply the weight of the drop by the percentage of collagen one is using, then divide by 10 and multiply by $10^{\wedge} 6$. Pour the mixture from the polypropylene centrifuge tubes into the sample cells and record the NTU reading using the turbidity meter.

Next, use $0.6 \%$ collagen and test $1,2,3,4$, and 5 drops at $0,3,5,10$, and 15 minutes in the refrigerated centrifuge at the same temperature and rpm as used 
before. Measure the weight of each drop and find the ppm using the method mentioned before. Pour the mixture from the polypropylene centrifuge tubes into the sample cells and record the NTU reading using the turbidity meter.

Then, use $0.9 \%$ collagen and test $1,2,3,4$, and 5 drops at $0,3,5,10$, and 15 minutes in the refrigerated centrifuge at the same temperature and $\mathrm{rpm}$ as used before. Measure the weight of each drop and find the ppm using the method mentioned before. Pour the mixture from the polypropylene centrifuge tubes into the sample cells and record the NTU reading using the turbidity meter.

\section{RESULTS}

The following is a selection of the turbidity readings and percent recovery from both the shake \& settle method and centrifuge method:

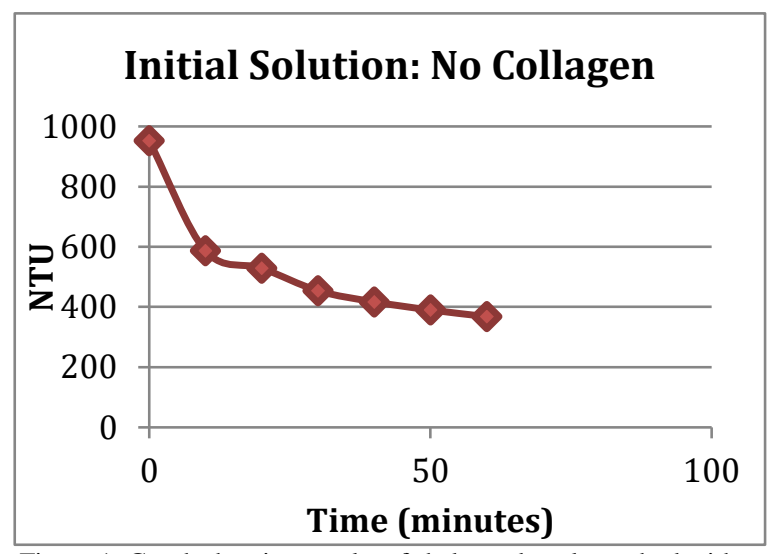

Figure 1: Graph showing results of shake and settle method with no addition of collagen

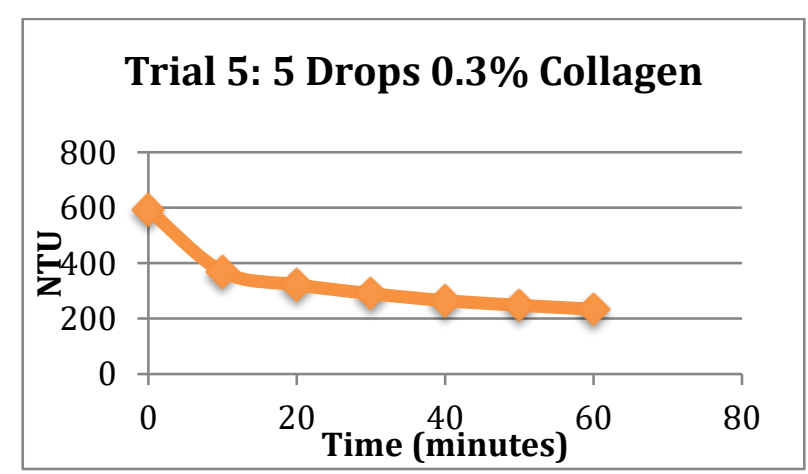

Figure 2: Graph showing results of shake and settle method with addition of 5 drops of $0.3 \%$ collagen

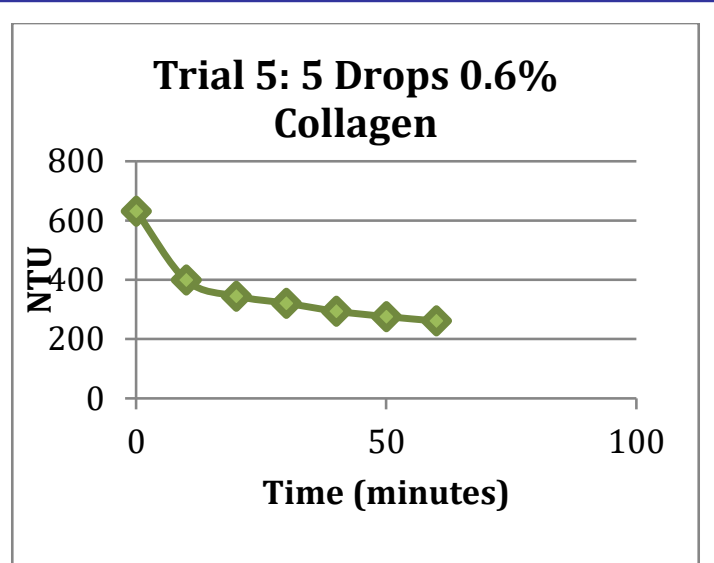

Figure 3: Graph showing results of shake and settle method with addition of 5 drops of $0.6 \%$ collagen

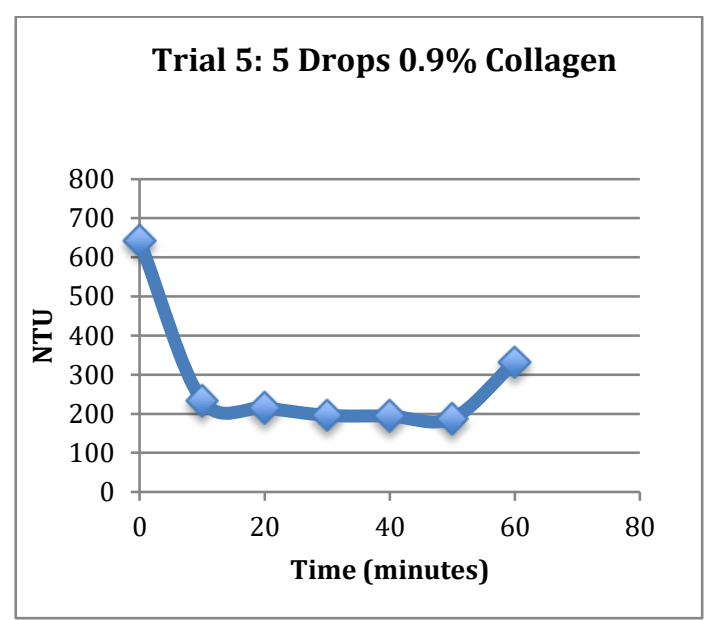

Figure 4: Graph showing results of shake and settle method with addition of 5 drops of $0.9 \%$ collagen

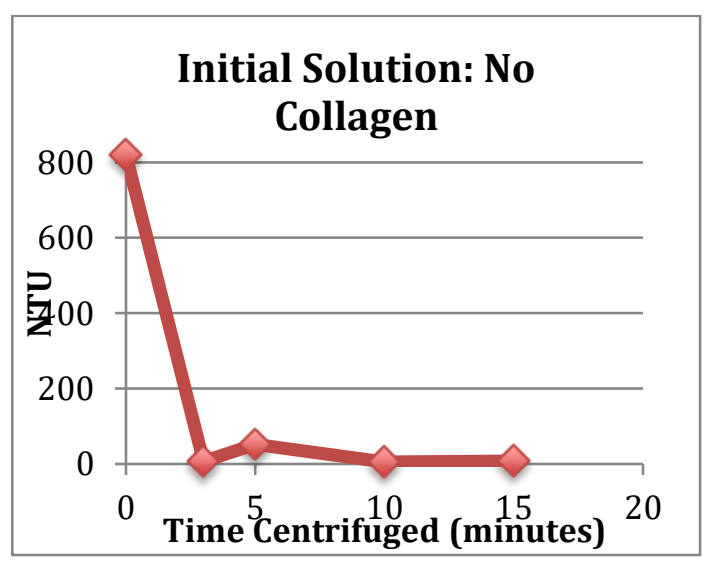

Figure 5: Graph showing results of centrifuge method with the addition of no collagen 


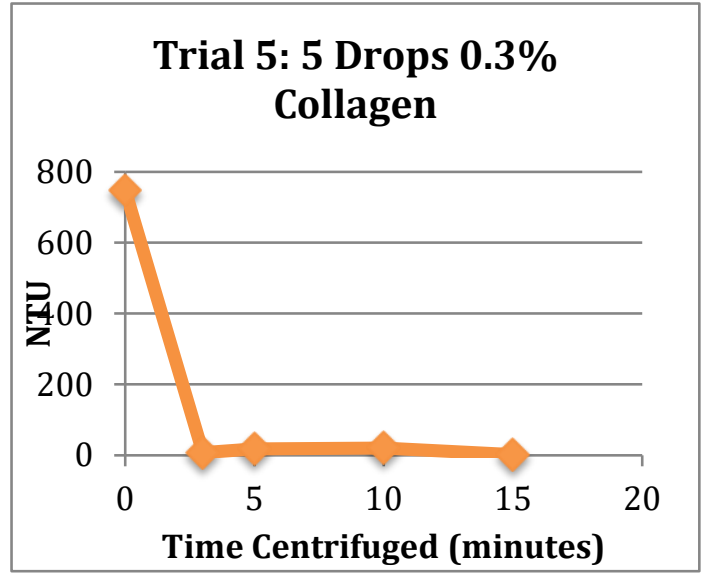

Figure 6: Graph showing results of centrifuge method with addition of 5 drops of $0.3 \%$ collagen

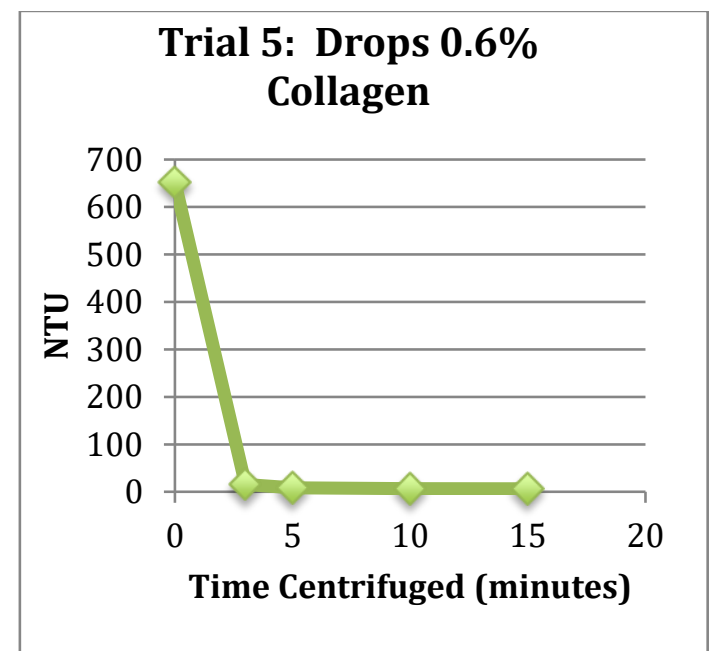

Figure 7: Graph showing results of centrifuge method with addition of 5 drops of $0.6 \%$ collagen

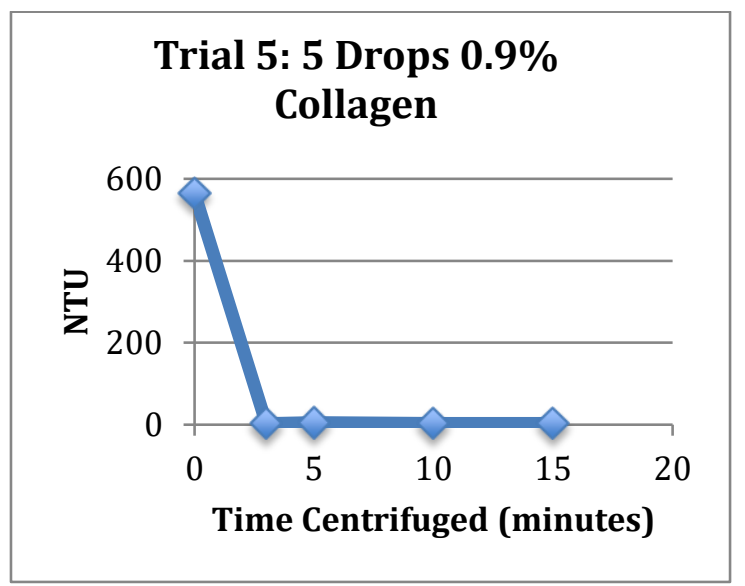

Figure 8: Graph showing results of centrifuge method with addition of 5 drops of $0.9 \%$ collagen

\section{DISCUSSION}

This research was conducted to experiment with alternate ways of cleaning turbid water since clean water is not easily found throughout the world. The methods used in this experiment are safer for humans as well as the environment. The results show that centrifuging rapidly decreases the NTU values as you increase the amount of time being centrifuged. After letting shake and settle test tubes sit for a few days, they still did not get as low of NTU values as centrifuged test tubes recorded.

In both experiments, the amount of drops did not affect the NTU values greatly at the initial times, however, as time went on, the more drops added, the greater the settling rates. We did not expect our initial NTU values to vary as much as they did between percentage of collagen and amount of drops. There was no consistent NTU value decrease as the percentages and amount of drops increased, as one would expect. The NTU value fluctuated or leveled out as time went on, which yielded inconsistent results. These inconsistencies could be due to human error, in which case, the experiment must be performed more than once to obtain accurate results.

When centrifuged, there was a dramatic decrease in NTU values between zero and three minutes no matter the percentage or amount of drops. In the shake \& settle method, there was also a decrease between zero minutes of settling time and the first ten minutes of settling, but the initial decrease was not as dramatic as it was for the centrifuged trials. The results show that adding more drops of collagen with a higher concentration will yield a lower turbidity. It was also found that centrifuging would cause too much disruption in the water, stopping the collagen fibers from interacting well with the kaolin particles. However, centrifuging for longer periods of time of at least an hour will increase the contact time between the kaolin particles and collagen fibers, yielding a lower turbidity reading.

\section{CONCLUSION}

It was shown that when using a kaolin/water solution, different amounts and concentrations of collagen had different effects on floc settling. This experiment provided environmentally friendly benefits as well as cost effective benefits. The shake and settle method costs less than centrifuging, but lower NTU values show that it is worth buying a refrigerated centrifuge. Both methods are more effective and cost efficient than chemical treatment and machinery currently in use.

\section{REFERENCES}

Maffia, Gennaro J. Collagen-based dispersions and macroporous structures. United States of America: Patent US6660829 B1. 9 December 2003. 